\title{
Dual-Space Adaptive Control of Redundantly Actuated Parallel Manipulators for Extremely Fast Operations With Load Changes
}

\author{
G. Sartori Natal, A. Chemori and F. Pierrot
}

\begin{abstract}
This paper deals with the dual-space adaptive control of $\mathbf{R} 4$ redundantly actuated parallel manipulator for applications with very high accelerations. This controller is compared experimentally with a dual-space feedforward controller (which may have good performances for specific cases, but has crucial losses of performance when there is any operational change (such as a change of load)), for a pickand-place task with accelerations of 30G (without payload) and 20G (with a payload of $200 \mathrm{~g}$ ). The objective of this paper is to show that the proposed dual-space adaptive controller not only keeps a very good performance independently of the operational case, but also has a better performance than the dual-space feedforward controller even when this last one is best configured to the given case.
\end{abstract}

\section{INTRODUCTION}

It is known that parallel manipulators have important advantages in comparison to serial manipulators in terms of speed/acceleration, stiffness, accuracy and payload. In order to deal with their main disadvantage (abundance in singularities in the workspace [1]), actuation redundancy was proposed in [2]. A degree of actuation redundancy of a parallel manipulator is the difference, represented by a positive integer, between the number of its actuators and its degrees-of-freedom (dof) [3]. Actuation redundancy might also be a way to reach higher accelerations or to improve the homogeneity of acceleration performance over the workspace (this was the basic conjecture in [4]). It can also allow for more safety in case of breakdown of individual actuators [5],[6].

In order to apply the vast control literature developed for serial counterparts to parallel manipulators with redundant actuation, there is a need to develop an efficient dynamical model for parallel manipulators [7]. A dynamics formulation that could be applied to redundant parallel manipulators was presented in [8]. Based on this formulation, redundant actuation was used to eliminate undesired singularity effects in parallel manipulators in [7],[9]. In these works, kinematic and dynamic control methods were satisfactorily implemented experimentally in the task space, where the effect of the redundant actuators to the end-effector motion can be fully considered [10]. Important and probably very perspective research results were achieved by Müller in [11] and [12]. In his recent works, he took profit from a general solution for the inverse dynamics of redundantly

This work is supported by ECHORD PRADA: an European project.

G. Sartori Natal, A. Chemori and F. Pierrot are with LIRMM, Univ. Montpellier 2 - CNRS, 161 rue Ada, 34392 Montpellier, France sartorinatalelirmm. fr actuated parallel manipulators to develop a computationallyefficient open-loop preload control scheme (depending on a single preload parameter), the simplicity of which makes it applicable in real-time control applications with backlash avoidance, especially for the cases of simply-redundant (i.e. one redundant actuator) parallel robots. In [13], in order to overcome the influences of modeling errors and nonlinear friction, a nonlinear computed torque control was introduced. In [14], a hybrid position/force adaptive control for redundantly actuated parallel manipulators has been proposed, and an adaptive controller for redundantly actuated parallel manipulator was designed in [10]. It is important to emphasize, however, that in none of these works the effect of load changes was analyzed (neither how the proposed controllers would deal with such operational changes).

In this paper, a dual-space adaptive controller is proposed (based on the adaptive control scheme proposed in [15]) in order to guarantee the best possible performance of a redundantly actuated parallel manipulator not only in an unloaded condition, but also when carrying a relevant payload at a very high speed.

This paper is organized as follows. In section II, a brief description of the R4 redundantly actuated parallel manipulator is presented. The proposed control scheme is detailed in section III. Section IV is devoted to the pick-and-place trajectory generation. The obtained experimental results are presented in section V. Conclusion and future works are discussed in section VI.

\section{R4 PARALLEL MANIPULATOR}

\section{A. Description of the R4 robot}

The R4 parallel manipulator (cf. Fig. 1) has the following main characteristics:

i) 3-dof (translations along $\mathrm{x}-\mathrm{y}-\mathrm{z}$ axis) and 4 actuators (redundantly actuated),

ii) Each motor has a maximum torque of $127 \mathrm{~N} . \mathrm{m}$,

iii) A workspace of at least a cylinder of $300 \mathrm{~mm}$ radius and $100 \mathrm{~mm}$ height.

Its geometrical parameters are summarized in table I and the reader is referred to [4] for their illustration.

\section{B. Simplified Direct Dynamics}

Some simplifications on the dynamics of the robot were made during its design phase in order to evaluate which configuration would be the optimal in terms of performance and cost, such as the neglect of joint friction, the inertia of the forearms and the gravity acceleration, as well as the consideration that the mass of the forearms is split in two 
parts (half of the mass is transferred to the end of the arm whereas the other half is transferred to the traveling plate). These assumptions are discussed in [4] and in [16].

TABLE I

GeOMETRIC/DyNAMics PARAMETERS

\begin{tabular}{|c|c|c|c|}
\hline$r_{b}[\mathrm{~m}]$ & $r_{t p}[\mathrm{~m}]$ & $l_{i}[\mathrm{~m}]$ & $L_{i}[\mathrm{~m}]$ \\
\hline 0.135 & 0.05 & 0.2 & 0.53 \\
\hline \hline$M_{t p}[\mathrm{~kg}]$ & $M_{\text {forearm }}[\mathrm{kg}]$ & $I_{\text {act }}\left[\mathrm{kg} \cdot \mathrm{m}^{2}\right]$ & $I_{\text {arm }}\left[\mathrm{kg} \cdot \mathrm{m}^{2}\right]$ \\
\hline 0.2 & 0.065 & 0.003 & 0.005 \\
\hline
\end{tabular}
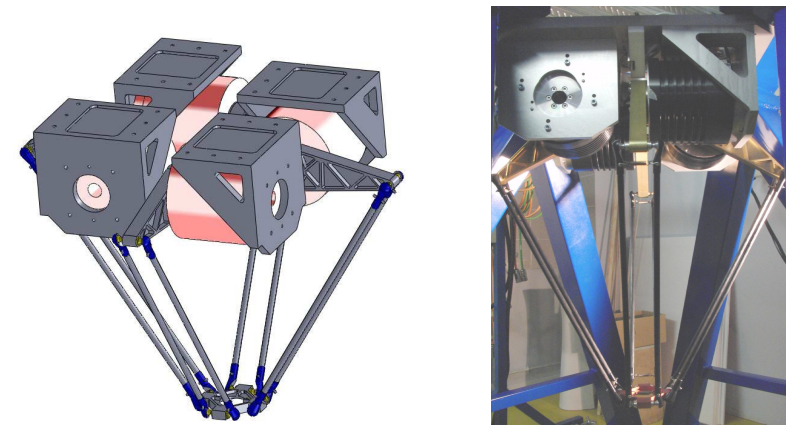

Fig. 1. The R4 parallel manipulator: Schematic view of the CAD design (left), side view of the robot prototype (right)

The expression of the simplified direct dynamic model of this robot is given by [4]:

$$
\ddot{x}=\left(M_{T}+J_{m}{ }^{T} I_{T} J_{m}\right)^{-1} J_{m}^{T}\left(\Gamma-I_{T} \dot{J}_{m} \dot{x}\right)
$$

where $\dot{x} \in \mathbb{R}^{m}$ and $\ddot{x} \in \mathbb{R}^{m}$ are the vectors of Cartesian velocities and accelerations; $M_{T}=\operatorname{Diag}\left\{M_{t p}+n \frac{M_{\text {forearm }}}{2}\right\}_{m \times m}$ $=M_{t o t} I_{m \times m}$ is a diagonal matrix with $m$ diagonal terms, being $M_{t p}$ the mass of the traveling plate, $M_{\text {forearm }}$ the mass of the forearm, $M_{t o t}$ the scalar value of the diagonal of $M_{T}, m$ the number of degrees-of-freedom $(m=3)$ and $n$ the number of motors $(n=4) ; I_{T}=\operatorname{Diag}\left\{I_{a c t}+I_{a r m}\right\}_{n \times n}=I_{t o t} I_{n \times n}$ is a diagonal matrix with $n$ diagonal terms, where $I_{a c t}$ and $I_{\text {arm }}$ are the inertia of the actuators and the inertia of the arms, respectively, and $I_{t o t}$ is the scalar value of the diagonal of $I_{T} ; J_{m} \in \mathbb{R}^{n \times m}$ and $\dot{J}_{m} \in \mathbb{R}^{n \times m}$ are the generalized inverse Jacobian matrix and its first derivative, respectively; and $\Gamma \in \mathbb{R}^{n}$ represents the torques generated by the actuators. For further details on the mechanical design of the R4 parallel manipulator, the reader is referred to [4]. After some algebraic manipulations, this dynamic model can be rewritten as:

$$
\Gamma=H^{T} M_{T} \ddot{x}+I_{T} \ddot{q}
$$

being $H$ the pseudo-inverse of $J_{m}$, that is $H=J_{m}^{+}=$ $\left(J_{m}^{T} J_{m}\right)^{-1} J_{m}^{T}$, and $\ddot{q}$ the vector of joint accelerations.

As mentioned in section I, the R4 parallel manipulator is redundantly actuated (4 actuators vs. 3-dof). This characteristic has important advantages in terms of mechanical capabilities of the robot, but in terms of control, new issues arise: not only classical joint control schemes are unable to deal with dynamic effects on the Cartesian space [3], but they are also unable to cope with the actuation redundancy (the integral term will be disturbed by kinematic inconsistencies). In order to deal with these issues, it is better to implement the control law in Cartesian space. It will be shown in the following that such a controller can easily be completed by a dual-space feedforward that has an effect similar to the computed torque controller.

\section{PROPOSED DUAL-SPACE CONTROL SCHEMES: A FEEDFORWARD CONTROLLER AND AN ADAPTIVE CONTROLLER}

\section{A. The dual-space feedforward controller}

The dual-space feedforward controller consists basically in a PID in the Cartesian space with a feedforward of both desired Cartesian/joint accelerations to improve the tracking performance. This control approach is illustrated through the block diagram of Fig. 2:

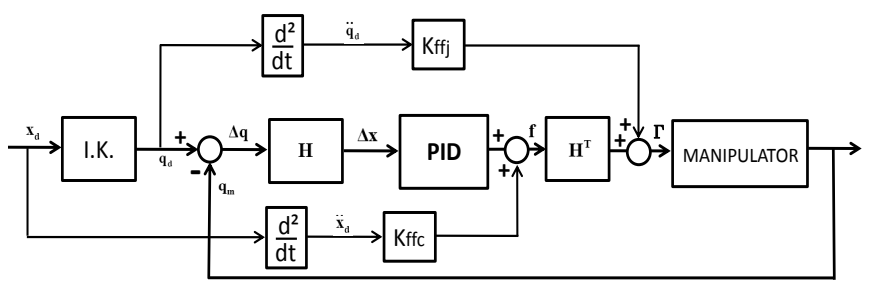

Fig. 2. Block diagram of the proposed dual-space feedforward controller

The desired trajectory $x_{d}$ is given in the Cartesian space. As only the joint positions $q_{m}$ are measured (the Cartesian positions are computed using the Forward Kinematic of the robot), this desired trajectory is converted to its counterpart $q_{d}$ in the joint space through the inverse kinematics (I.K. block in Fig. 2) of the robot [4], such that the corresponding tracking error can be obtained. The joint tracking errors $\Delta q$ must then be reconverted to the Cartesian space $\Delta x$ in order to be used by the PID controller. As the joint tracking errors are assumed to be significantly small, and the sampling time $\Delta t$ is of only $0.1 \mathrm{~ms}\left(10^{-4} s\right)$, let $\frac{\Delta q}{\Delta t} \simeq \frac{d q}{d t}$. Then, it is recalled that $\dot{q}=J_{m} \dot{x}$, being $\dot{q}$ the joint velocity, $\dot{x}$ the Cartesian velocity of the platform and $J_{m}$ the generalized inverse Jacobian matrix, as defined in [4]. This relation has a unique solution if $J_{m}$ is square, however in the case of redundantly actuated manipulators $(n>m)$, the inverse relation will have infinite solutions. In order to cope with this issue, the pseudo-inverse of $J_{m}$ is used instead (the pseudo-inverse has the property of generating a solution with the minimum Euclidian norm). Therefore, one has that $\dot{x}=H \dot{q}$, where $H$ is the pseudoinverse of $J_{m}$, that is $H=J_{m}^{+}=\left(J_{m}{ }^{T} J_{m}\right)^{-1} J_{m}{ }^{T}$. The following relation between the joint errors $\Delta q$ and the Cartesian errors $\Delta x$ can then be obtained:

$$
\frac{d x}{d t}=H \frac{d q}{d t} \Rightarrow \frac{\Delta x}{\Delta t} \simeq H \frac{\Delta q}{\Delta t} \Rightarrow \Delta x \simeq H \Delta q
$$

By straightforward analysis of Fig. 2, the proposed dualspace feedforward control law is given by: 


$$
\Gamma=H^{T} f+K_{f f j} \ddot{q}_{d}
$$

being $f=K_{p} e+K_{i} \int e(t) d t+K_{d} \frac{d e(t)}{d t}+K_{f f c} \ddot{x}_{d}$ the force applied on the traveling plate, $e=\Delta x, K_{p}, K_{i}$ and $K_{d}$ the Cartesian PID gains, $K_{f f c}$ the Cartesian acceleration feedforward gain and $K_{f f j}$ is the joint acceleration feedforward gain. In order to compensate for the effects of the dynamics of the system, it is possible to see from the simple rewritten dynamic model (2) that the feedforward gains that should multiply $\ddot{x}_{d}$ and $\ddot{q}_{d}$ are simply the system parameters $\left(K_{f f c}=\right.$ $M_{t o t}$ and $\left.K_{f f j}=I_{t o t}\right)$. For information purposes, the gains of the PID in the Cartesian space was tuned experimentally through the procedure of small steps and the feedforward gains were tuned manually until the best performances could be achieved for the case with and without payload. These gains are presented in table II.

\section{B. The dual-space adaptive controller}

The proposed dual-space adaptive controller is based on the adaptive control scheme proposed in [15]. The most important characteristic of this controller is its capability of taking into consideration the dynamics of the system and estimate its parameters automatically in real-time. Considering the rewritten dynamics of the system (2), we propose the implementation of this controller in dual-space, being its general expression given as follows:

$$
\Gamma=\hat{M}(q) \ddot{q}_{d}+\hat{C}(q, \dot{q}) \dot{q}_{d}+\hat{G}(q)+K_{p} e+K_{d} \dot{e}
$$

where $e=q_{d}-q, \hat{M}, \hat{C}, \hat{G}$ are the estimates of $M, C$ and $G$ (being $M(q) \in \mathbb{R}^{n \times n}$ the inertia matrix, $C(q, \dot{q}) \in \mathbb{R}^{n \times 1}$ the vector of Coriolis and centrifugal forces, and $G(q)$ the gravity vector), respectively. Considering the dynamic model of R4 manipulator, the following control law is then proposed:

$$
\Gamma=H^{T} \hat{M}_{T} \ddot{x}_{d}+\hat{I}_{T} \ddot{q}_{d}+K_{p} e+K_{d} \dot{e}
$$

which can be rewritten in the Cartesian space as:

$$
F=Y_{r} \hat{\theta}+K_{p} e_{c}+K_{d} \dot{e}_{c}
$$

where $K_{p}$ and $K_{d}$ are positive gains, $e_{c}=x_{d}-x, \dot{e}_{c}=\dot{x}_{d}-\dot{x}$, and:

$$
Y_{r}=\left[\begin{array}{ll}
I_{3 \times 3} \ddot{x}_{d} & J_{m}^{T} I_{4 \times 4} \ddot{q}_{d}
\end{array}\right] ; \quad \hat{\theta}=\left[\begin{array}{c}
\hat{M}_{t o t} \\
\hat{I}_{t o t}
\end{array}\right]
$$

being $Y_{r}$ and $\hat{\theta}$ the regressor vector and the vector of estimated parameters, respectively. These estimated parameters vary according to the following adaptation rule $(i=1,2)$ :

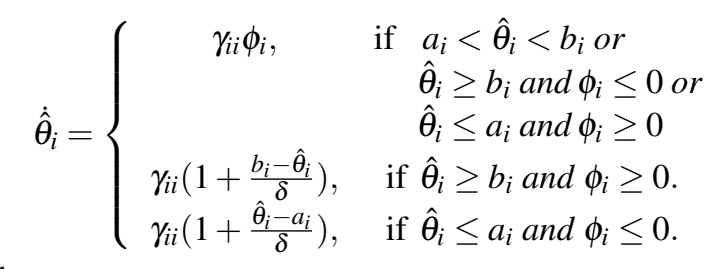

where

- $\gamma_{i i}$ is the $i^{t h}$ element of the diagonal adaptation gain matrix $\gamma$,
- $\hat{\theta}_{i}$ represents the estimates of each unknown parameter,

- $a_{i}$ and $b_{i}$ are the lower and upper bounds of each estimative, respectively,

- $\phi_{i i}$ is the $i^{t h}$ element of the column matrix $\phi=-Y_{r}^{T} s$; being $s=\dot{e}_{c}+\lambda e_{c}$, where $\lambda=\frac{\lambda_{0}}{1+\|e\|}$, being $\lambda_{0}$ and $\delta$ positive constants.

The chosen adaptive gains were $\gamma_{11}=0.2$ and $\gamma_{22}=$ $1.5 \times 10^{-4}$. In order to decide the good range for the estimated parameters, the behavior of the feedforward controller with relation to its best gains for each specific case were taken into account. Considering that the best $K_{f f c}$ gains of the feedforward controller were 0.625 for the case without payload and 0.825 for the case with a payload of $200 \mathrm{~g}$, and also considering that bigger payloads may be used in future experiments, the chosen range for the $\hat{M}_{t o t}$ parameter (in $\mathrm{kg}$ ) was of $[0.525 ; 1]$, which means $a_{1}=0.525$ and $b_{1}=1$. Concerning the inertia $\hat{I}_{t o t}$ parameter (in $\mathrm{kg} \cdot \mathrm{m}^{2}$ ), which is equivalent to the $K_{f f j}$ feedforward gain, the range was chosen as [0.006;0.018], which means $a_{2}=0.006$ and $b_{2}=0.018$. In this paper, only the behavior of the $\hat{M}_{t o t}$ parameter will be detailed, as this is the parameter that directly compensates for the load changes. The chosen parameters of the dual-space adaptive controller are summarized in table III.

\section{TRAJECTORY GENERATION: 3D PICK-AND-PLACE MOVEMENTS}

The objective of this case study is to evaluate the capability of the proposed control approach to deal with very high accelerations in a pick-and-place like trajectory. This kind of trajectory is largely used in the industry for many different applications, such as food/medicaments packaging, lasercutting, electronic components assembly, etc.

In this case study, the desired trajectory is chosen such that movements of different distances would have to be performed in the same amount of time, which would require different accelerations for each one of them, demonstrating the good applicability of the proposed control scheme. The sequence of movements to be executed in this case study is the following (as illustrated in Fig. 3):

1) Pick 1 - Place 1: From $(-0.1,0.1) \mathrm{m}$ to $(0.1,-0.1) \mathrm{m}$,

2) Place 1 - Pick 2: From $(0.1,-0.1) \mathrm{m}$ to $(0.1,0.1) \mathrm{m}$,

3) Pick 2 - Place 2: From $(0.1,0.1) \mathrm{m}$ to $(-0.1,-0.1) \mathrm{m}$,

4) Place 2 - Pick 1: From $(-0.1,-0.1) \mathrm{m}$ to $(-0.1,0.1) \mathrm{m}$.

Each movement was performed in $0.08 s$ without payload ( $0.32 s$ for the whole cycle), and in $0.1 s$ with payload $(0.4 s$ for the whole cycle). Their maximum height was equal to $2.5 \mathrm{~cm}$. The trajectory generation algorithm used in this case is based on a polynomial interpolation of degree five [17]. This algorithm guarantees the continuity of the movement in position, velocity and also in acceleration.

\section{REAL-TIME EXPERIMENTAL RESULTS}

In this section, real-time experimental results obtained by the application of the proposed control schemes described in section III on the parallel manipulator R4 described in section II in order to track the reference trajectories detailed 


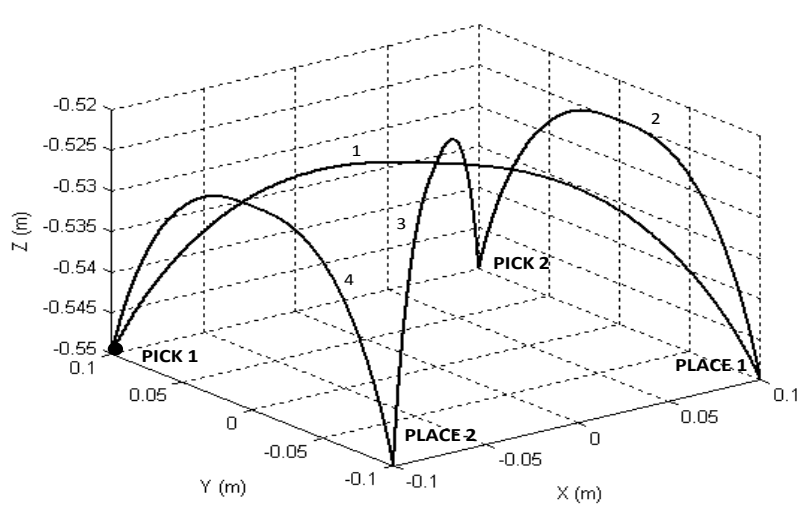

Fig. 3. Isometric view of the 3D pick-and-place trajectory

in section IV (firstly with a payload of $200 \mathrm{~g}$ at $20 \mathrm{G}$ of acceleration and then without payload at 30G) are presented and discussed.

TABLE II

Parameters of the Dual-Space Feedforward Controller

\begin{tabular}{|c|c|c|c|c|}
\hline$K_{p}$ & $K_{i}$ & $K_{d}$ & $K_{f f c}$ & $K_{f f j}$ \\
\hline 8000 & 600 & 40 & 0.825 & 0.012 \\
\hline
\end{tabular}

TABLE III

Configuration of the DUAL-Space Adaptive Controller

\begin{tabular}{|c|c|}
\hline Adaptive gains & $\gamma_{11}=0.2 / \gamma_{22}=1.5 e^{-4}$ \\
\hline Range of $\hat{M}_{\text {tot }}(\mathrm{kg})$ & {$[0.525 ; 1]$} \\
\hline Range of $\hat{I}_{\text {tot }}\left(\mathrm{kg} \cdot \mathrm{m}^{2}\right)$ & {$[0.006 ; 0.018]$} \\
\hline$\lambda_{0}$ & 100 \\
\hline$\delta$ & 0.0001 \\
\hline$K_{p}$ & 8000 \\
\hline$K_{d}$ & 40 \\
\hline
\end{tabular}

\section{A. The experimental testbed}

The proposed control schemes were implemented in Simulink/Matlab of Mathworks, being compiled using XPC Target real-time toolbox, and uploaded to a target PC, which managed the real-time task execution with a sampling frequency of $10 \mathrm{KHz}$.

In the following experiment, the robot goes from the rest position to the desired initial position $(-0.1,0.1,-0.55) m$ and then executes two cycles of the proposed pick-and-place trajectory while carrying a payload of $200 \mathrm{~g}$.

\section{B. 3D pick-and-place movements with a payload of $200 \mathrm{~g}$ at $20 G$}

In this scenario, the adaptive controller is compared to the feedforward controller (configured with its best value of $K_{f f c}$ for the case with a payload of $\left.200 \mathrm{~g}\left(K_{f f c}=0.825\right)\right)$ at $20 \mathrm{G}\left(\approx 200 \mathrm{~m} / \mathrm{s}^{2}\right)$ of acceleration. The objective of this experiment is to show that the feedforward controller may have a good performance when configured with its best value of $K_{f f c}$ for a specific scenario, but even in this case it
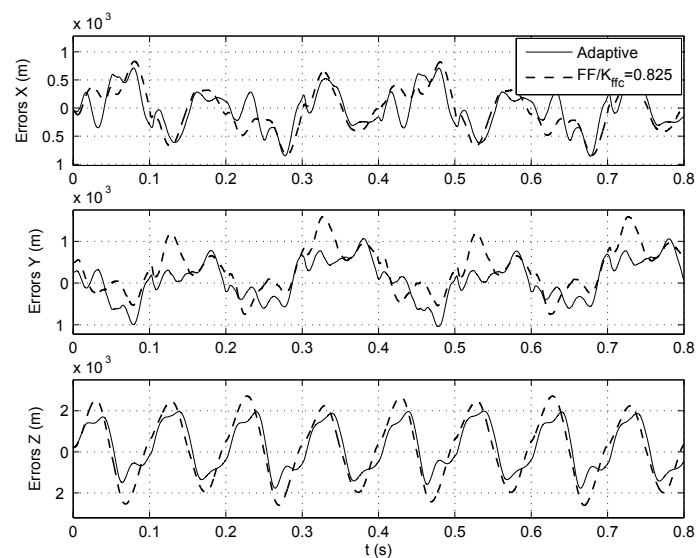

Fig. 4. 3D pick-and-place tracking errors with a payload of $200 \mathrm{~g}$ for an acceleration of $20 \mathrm{G}$

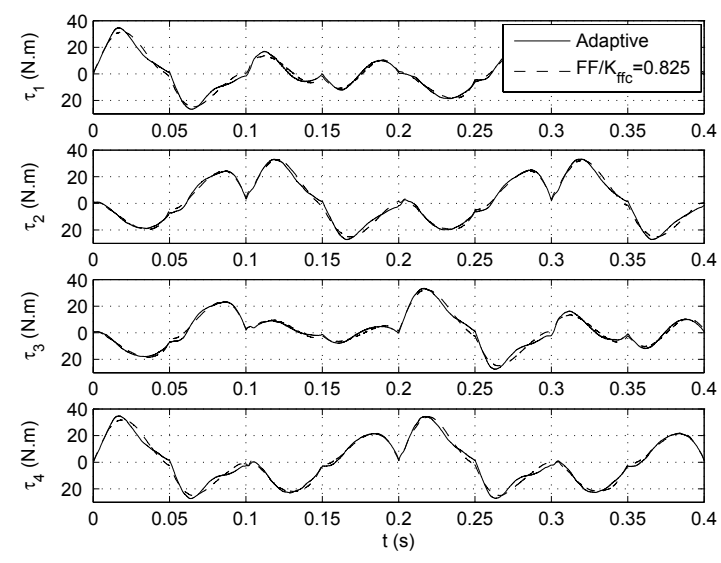

Fig. 5. Torques applied by the 4 motors with a payload of $200 \mathrm{~g}$ for an acceleration of $20 \mathrm{G}$

will remain worse than the adaptive controller. The obtained results for this scenario are depicted in Figs. 4-5.

By analyzing Fig. 4, it is possible to notice that the adaptive controller is able to provide a better overall tracking performance than the feedforward controller even with its best value of $K_{f f c}$ for this case. For the X-Y axes, even though the feedforward has provided a good performance, the adaptive controller was able to keep the tracking errors inside a smaller interval, as shown in table IV. For the Z-axis, the difference between the performances of both controllers is bigger and easily visible. The superior performance of the adaptive controller in this case is further confirmed by the Root Mean Square Errors (RMSE) presented in table IV. This RMSE takes into consideration the Root Mean Square Errors of all axes equally, as detailed in the following:

$$
e_{r m s}=\sqrt{e_{r m s_{x}}^{2}+e_{r m s_{y}}^{2}+e_{r m s_{z}}^{2}}
$$

The torques generated by each controller are presented in Fig. 5, which demonstrates that the adaptive controller 

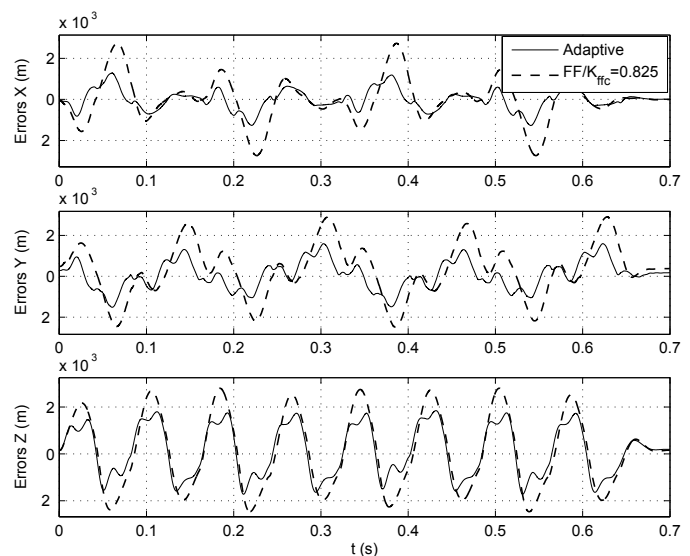

Fig. 6. 3D pick-and-place tracking errors without payload for an acceleration of $30 \mathrm{G}$
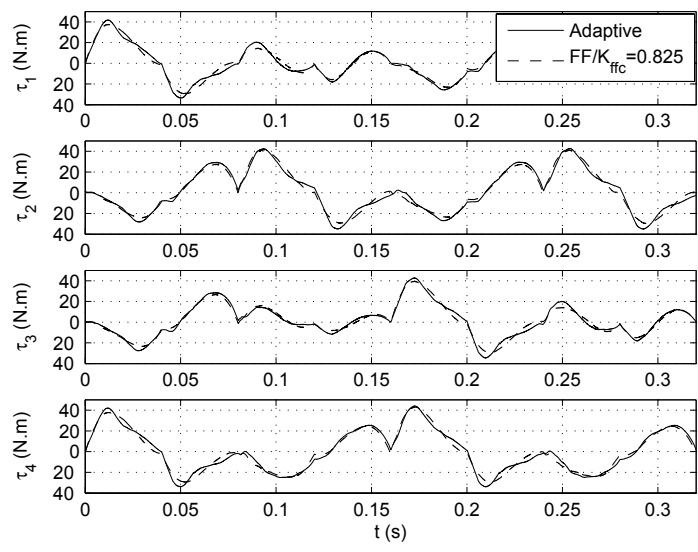

Fig. 7. Torques applied by the 4 motors without payload for an acceleration of $30 \mathrm{G}$

generated a signal with a slightly bigger amplitude than the feedforward controller.

In the next scenario, it will be shown that the feedforward controller loses much performance when not having an adequate manual update of $K_{f f c}$ for a different case, while the adaptive controller maintains its very good performance independently of the scenario without any need of manual adjustments of its parameters.

\section{3D pick-and-place movements without payload at 30G}

In this scenario, the robustness of the adaptive controller to load changes and the lack of robustness of the feedforward controller to load changes are confirmed. From Fig. 6, it is possible to notice that the feedforward controller, when not manually updated to a new scenario, has a crucial loss of performance (more than 100\% peak-to-peak (difference between the highest peak and the lowest valley of a signal) error increase in the X-Y axes in this case), which shows that it is not robust to operational changes. As in the previous scenario, the adaptive controller maintains its very good
TABLE IV

TRACKING PERFORMANCE OBTAINED WITH THE PROPOSED CONTROLLERS FOR A 20G PICK-AND-PLACE TRAJECTORY WITH A PAYLOAD OF $200 \mathrm{~g}$

\begin{tabular}{|c|c|c|}
\hline Performance & Adaptive & FF $\left(K_{f f c}=0.825\right)$ \\
\hline Error peaks $(\mathrm{X}-\mathrm{Y})$ & {$[-1,1] \mathrm{mm}$} & {$[-0.75,1.6] \mathrm{mm}$} \\
\hline Error peaks $(\mathrm{Z})$ & {$[-1.77,2] \mathrm{mm}$} & {$[-2.6,2.7] \mathrm{mm}$} \\
\hline RMSE & $1.33 \mathrm{~mm}$ & $1.71 \mathrm{~mm}$ \\
\hline Control Signals & \multicolumn{2}{|c|}{ Smooth/far from limits } \\
& \multicolumn{2}{|c|}{ Adaptive controller: Slightly bigger amplitude } \\
\hline
\end{tabular}

performance without any need of manual adjustment of its parameters. Table IV shows that the adaptive controller maintained an overall similar performance with relation to the previous case (especially for the RMSE that remained nearly unchanged) while the feedforward controller had critical losses, which would only be compensated by an adequate manual adjustment of the $K_{f f c}$ gain. Fig. 7 confirms that the adaptive controller generated a control signal with a slightly bigger amplitude than the feedforward controller. These experiments have been registered on the accompanying video.

TABLE V

TRACKING PERFORMANCE OBTAINED WITH THE PROPOSED CONTROLLERS FOR A 30G PICK-AND-PLACE TRAJECTORY WITHOUT PAYLOAD

\begin{tabular}{|c|c|c|}
\hline Performance & Adaptive & FF $\left(K_{f f c}=0.825\right)$ \\
\hline Error peaks $(\mathrm{X}-\mathrm{Y})$ & {$[-1.51,1.6] \mathrm{mm}$} & {$[-2.73,2.9] \mathrm{mm}$} \\
\hline Error peaks $(\mathrm{Z})$ & {$[-1.72,1.84] \mathrm{mm}$} & {$[-2.26,2.77] \mathrm{mm}$} \\
\hline RMSE & $1.4 \mathrm{~mm}$ & $2.36 \mathrm{~mm}$ \\
\hline Control Signals & \multicolumn{2}{|c|}{ Smooth/far from limits } \\
& Adaptive controller: Slightly bigger amplitude \\
\hline
\end{tabular}

\section{Evolution of the estimated parameter $\hat{M}_{t o t}$}

In order to compensate for the load changes, the parameter $M_{\text {tot }}$ was estimated in real-time by the adaptive controller. The feedforward gain $K_{f f c}$, which will be considered as an offline estimation of $M_{t o t}$, remains constant during all the experiments. The behavior of both estimations of $M_{t o t}$ is depicted in Figs. 8-9 for different accelerations.

For the first scenario, the value of $K_{f f c}$ which provides the best performance of the feedforward controller is equal to 0.825 (dashed curves in both Figs. 8-9). The first thing to be mentioned is that the convergence of the estimation of the adaptive controller from the initial value to a region around 0.825 is fast enough to be accomplished before the first stop point is reached (which is the expected performance in a pick-and-place task, where the robot will perform movements with payload followed by movements without payload). This confirms the fact that the tracking performance of the adaptive controller will barely be affected by an initial value which is different from the best value for the specific case, and also justifies the good performance of the feedforward controller when keeping this value constant during this experiment.

For the second scenario, the crucial loss of performance of the feedforward controller is justified. In Fig. 9, it is 

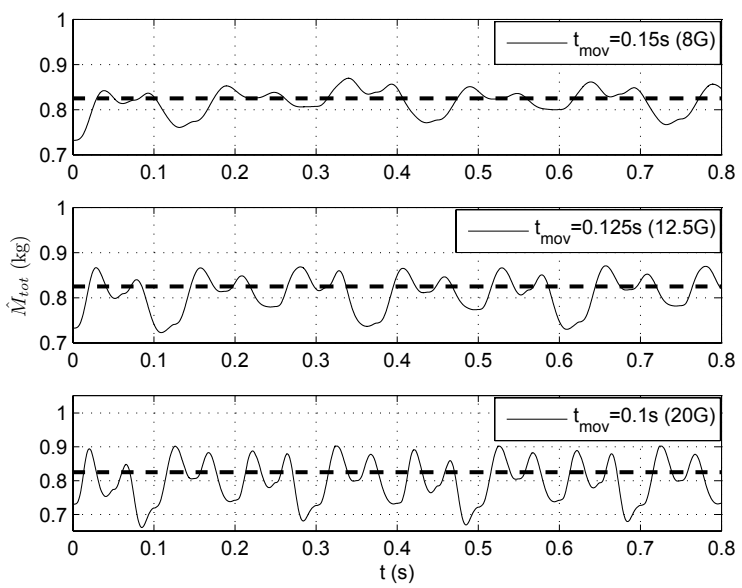

Fig. 8. Evolution of the parameters $\hat{M}_{t o t}$ and $K_{f f c}$ (dashed) for different accelerations (with a payload of $200 \mathrm{~g}$ )
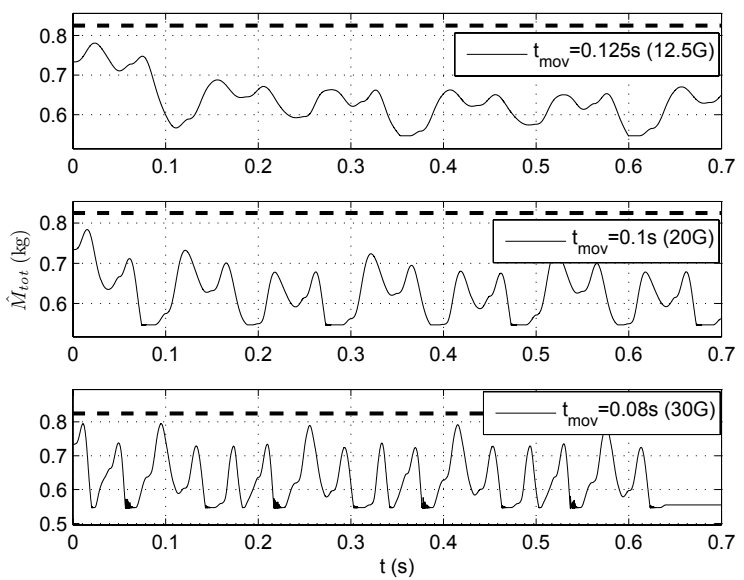

Fig. 9. Evolution of the parameters $\hat{M}_{t o t}$ and $K_{f f_{c}}$ (dashed) for different accelerations (without payload)

shown that when not manually updating the feedforward gain $K_{f f c}$, this estimation will now remain with an inadequate value. The estimation of the adaptive controller converges to a region around $\hat{M}_{t o t}=0.625$, which is the best value of $K_{f f c}$ for this case. Another point to be mentioned is the increased oscillations in parameter estimation with the increase of acceleration. Between the most reasonable causes, one can mention the increase of unmodeled dynamics effects (such as the dry and viscous frictions, counter-electromotice forces, etc.), which become more important with higher accelerations, thus becoming a relevant disturbance source. However, the robustness of the adaptive controller enables it to maintain both smoothness and good performance of the closed-loop system in terms of tracking, as well as in terms of evolution of the control inputs (the same general form for both controllers, without addition of oscillations by the adaptive controller, cf. Figs. 5,7), despite oscillations on parameters.

\section{CONCLUSION AND FUTURE WORKS}

In this paper, in order to guarantee the best possible tracking performance of the redundantly actuated parallel manipulator R4 independently of the operational scenario, a dual-space adaptive controller was proposed. For evaluation purposes, it was compared with a dual-space feedforward controller, which is able to provide a good performance when best configured for a specific case, but loses much performance when any operational change occurs. The realtime experimental results show clearly that the proposed adaptive controller is able to compensate for the load changes quickly enough to be applied on a pick-and-place task, even with very high accelerations. As future works, the addition of the friction in the dynamic model will be analyzed, such that a corresponding adaptation term can be added in the adaptive controller, and other applications such as laser cutting with complex trajectories shall be performed.

\section{REFERENCES}

[1] S. Kock and W. Schumacher, "A parallel x-y manipulator with actuation redundancy for high-speed and active-stiffness applications," Proc. IEEE Conf. Robotics Automat., pp. 2295-2300, 1998.

[2] F. C. Park and J. W. Kim, "Singularity analysis of closed kinematic chains," ASME Trans. J. Mech. Des., vol. 121, no. 1, pp. 32-38, 1999.

[3] L. Ganovski, "Modeling, simulation and control of redundantly actuated parallel manipulators," PhD. dissertation, Université Catholique de Louvain, Faculté des Sciences Appliquées, Belgique, 2007.

[4] D. Corbel, M. Gouttefarde, O. Company and F. Pierrot, "Towards $100 \mathrm{~g}$ with $\mathrm{pkm}$. is actuation redundancy a good solution for pick-andplace?," Proc. of the IEEE Int. Conf. on Rob. and Autom., pp. 46754682, 2010.

[5] Y. Yi, J. E. Mcinroy and Y. X. Chen, "Fault tolerance of parallel manipulators using task space and kinematic redundancy," IEEE Trans. Robotics, vol. 22, no. 5, pp. 1017-1021, 2006.

[6] R. G. Roberts, H. G. Yu and A. A. Maciejewski, "Fundamental limitations on designing optimally fault-tolerant redundant manipulator," IEEE Trans. Robotics, vol. 24, no. 5, pp. 1224-1237, 2008.

[7] H. Cheng, Y. K. Yiu and Z. X. Li, "Dynamics and control of redundantly actuated parallel manipulators," IEEE Trans. Mechatronics, vol. 8, no. 4, pp. 483-491, 2003.

[8] Y. Nakamura and M. Ghodoussi, "Dynamics computation of closedlink robot mechanisms with nonredundant and redundant actuators," IEEE Trans. on Robotics Automat., vol. 5, pp. 294-302, 1989.

[9] G.F. Liu, Y.L. Wu, X.Z. Wu, Y.Y. Kuen and Z.X. Li, "Analysis and control of redundant parallel manipulators," Proc. IEEE Int. Conf. Robot. Autom., pp. 3748-3754, 2001.

[10] W. W. Shang and S. Cong, "Nonlinear adaptive task space control for a 2-dof redundantly actuated parallel manipulator," Nonlinear Dynamics, vol. 59 , no. $1,2010$.

[11] A. Müller, "Control of simply-redundant full-actuated parallel manipulators," ECCOMAS Thematic Conference Multibody Dynamics, pp. $215-223,2005$.

[12] A. Müller, "Internal preload control of redundantly actuated parallel manipulators," Proc. IEEE Int. Conf. Robot. Autom., pp. 948-953, 2005.

[13] W. W. Shang and S. Cong, "Nonlinear computed torque control for a high-speed planar parallel manipulator," Mechatronics, vol. 19, no. 6, pp. 987-992, 2009.

[14] S. Hui, W. Xu-Zhang, L. Guan-Feng and L. Ze-Xiang, "Hybrid position/force adaptive control of redundantly actuated parallel manipulators," Acta Automatica Sinica, vol. 29, no. 4, pp. 567-572, 2003.

[15] K.W. Lee and H.K. Khalil, "Adaptive output feedback control of robot manipulators using high-gain observer," Int. J. of Contr., pp. 869-886, 1997.

[16] V. Nabat, "Robots parallèles à nacelle articulée - du concept à la solution industrielle pour le pick-and-place," $P h D$. dissertation, Université Montpellier II, Montpellier, France, 2007.

[17] W. Khalil and E. Dombre, Modeling, identification and control of robots. Butterworth-Heinemann, 2004. 